\title{
Китай в глобальных цепочках стоимости: некоторые факты
}

\author{
Наталья Волгина, Лю Пэнфэй * \\ Российский университет дружбы народов, Москва, Россия
}

Информация о статье

Поступила в редакичию: 23.09.2020

Принята

к опубликованию:

10.12.2020

УДК 339

JEL F140

\section{Ключевые слова:}

мировая торговля, Китай, глобальные цепочки стоимости, внутренняя добавленная стоимость, зарубежная добавленная стоимость (ЗДС), деглобализация

\section{Keywords:}

world trade, China, global value chains (GVCs), domestic value added (DVA), foreign value added (FVA), deglobalization

\begin{abstract}
Аннотация
В статье представлены особенности участия Китая в глобальной цепочке создания стоимости (далее - ГЦС) по сравнению с крупнейшими мировыми экспортерами. В соответствии с расчетами на основе статистической базы ОЭСР TiVa, авторы приходят к следуюшим выводам: в последние годы для всех стран было характерно снижение интегращии в ГЦС, при этом, показатели этого снижения для Китая были минимальными; сокращение участия стран в ГЦС напрямую связана с уменьшением доли зарубежной добавленной стоимости в экспорте, причем зависимость Китая от импортируемьх промежуточных продуктов снижалась наиболее быстрыми темпами.
\end{abstract}

\section{China in the Global Value Chains: Some Facts}

Natalia Volgina, Liu Peng-fei

\section{Abstract}

China's accession to the WTO has not only entailed China's increased role in the world trade, but has also symbolized the beginning of China's accelerated integration into the global value chains (GVCs). Initially, China was of interest to developed countries as a location with relatively cheap labor, but gradually its role in the GVC changed, and China became the largest exporter of intermediate goods of final demand in the industrial supply chains, primarily in the automotive and electronics sectors. Thus, it is necessary to evaluate Chinese exports not only and not so much in terms of gross figures, but in terms of value added indicators. The purpose of this work is to evaluate the features of China's participation in GVCs compared with the world's largest exporters such as the USA and Germany. In accordance with the calculations based on the OECD TiVa statistical database, the authors come to the following conclusions: all countries have experienced a decline in GSC integration in recent years, while the rate of the decline for China has been minimal; the decline in countries' participation in GSCs is directly related to the declining share of foreign value added in exports, with China's dependence on imported intermediate products declining most rapidly; at the same time China has increased its share of indirect domestic value added in its exports of intermediate goods by building up its own supply chain. Despite the fact that the countries participation in GVCs is declining, it remains very high, and it is too early to talk about the established trends in de-globalization.

* Автор для связи: lynvb@yandex.ru

DOI: https://dx.doi.org/10.24866/2311-2271/2020-4/20-27 


\section{Введение}

Вступление Китая в ВТО в 2001 г. (на очень выгодных для страны условиях) внесло изменения в структуру мировой экономики, ознаменовав начало быстрого роста доли Китая в мировой торговле. В 2018 г. доля Китая в мировом экспорте превысила 13\% (рис.1), тогда как в 2000 г. она не достигала и 4\%. При этом доля США в мировом экспорте 2018 г. составила 9\%, а всех стран ЕС - около 15\%.

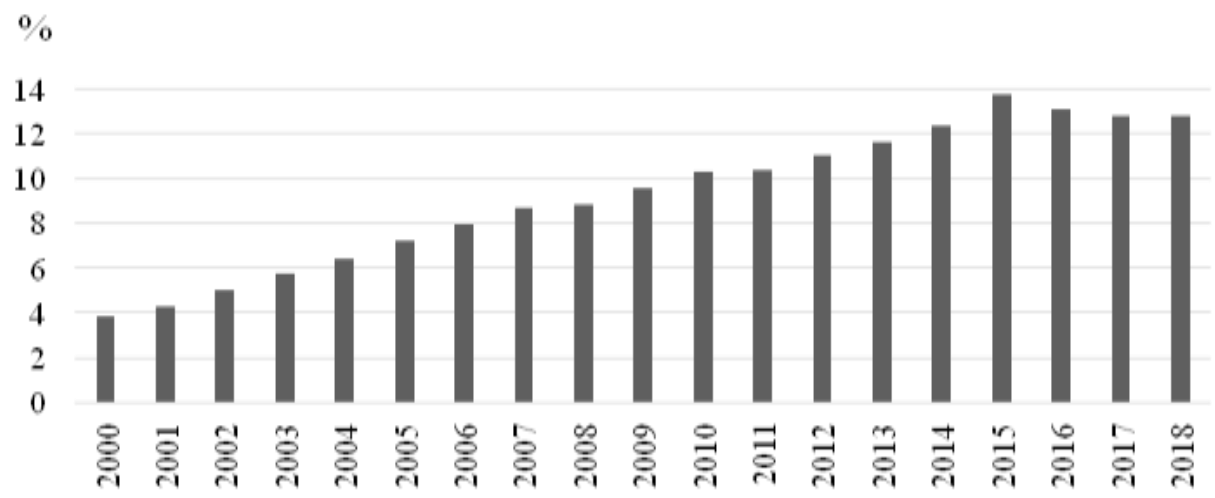

Рuc. 1. Доля Китая в мировом экспорте, 2000-2018 гг. (\%) Источник: рассчитано по данным ВТО

Если посмотреть на промышленный экспорт, то усиление роли Китая становится еще более очевидным (рис. 2): в настоящее время на его долю приходится около 19\% мирового экспорта товаров обрабатывающей промышленности, тогда как в 2000 г. эта доля составляла менее 5\%. Это явилось также результатом проведения правительства промышленной политики, нацеленной на стимулирование инноваций, которая была закреплена в программном документе «Сделано в Китае до 2025», опубликованном в 2015 г. КНР решила сосредоточиться на приоритетном развитии собственных технологий, ослабить зависимость от западных (в первую очередь, американских технологий). Китай ставит перед собой задачу добиться мирового лидерства в технологиях к 2049 г.

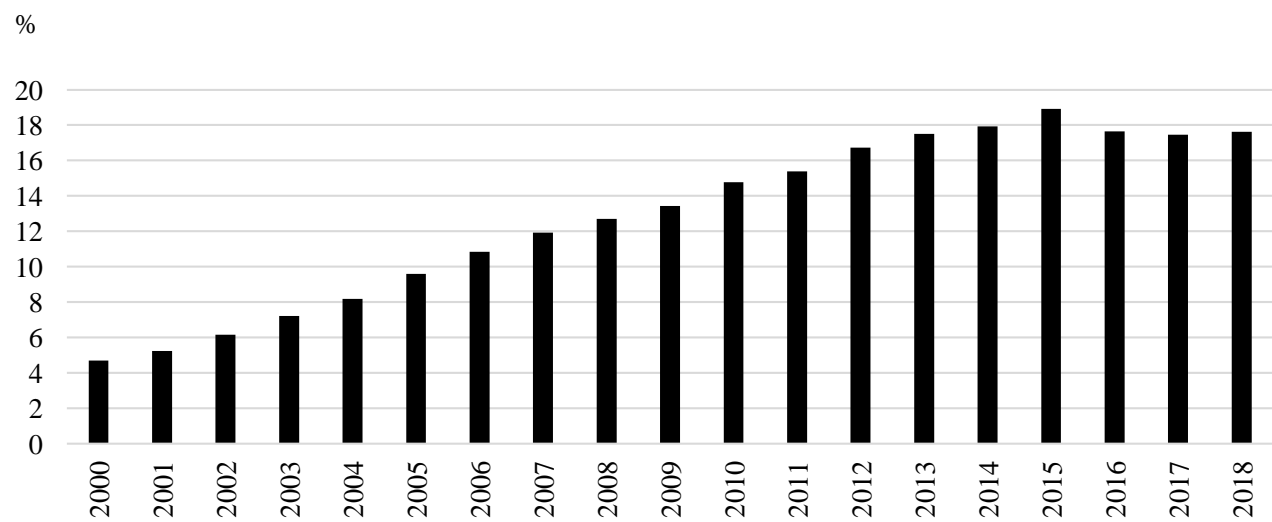

Рuc. 2. Доля Китая в мировом промышленном экспорте, 2000-2018 гг. (\%) Источник: рассчитано по данным ВТО

Следует отметить, что вступление Китая в ВТО повлекло за собой не только усиление роли страны в мировой торговле, но символизировало начало ускоренной интеграции в ГЦС [1]. Первоначально страна представляла интерес для развитых стран как локация с относительно дешевой рабочей силой, однако 
постепенно его роль в ГЦС менялась, и Китай превратился в крупнейшего экспортера промежуточных товаров финального спроса в промышленных цепочках поставок, в первую очередь, в автомобилестроении и электронике.

Итак, доля Китая в мировом валовом экспорте составляет весьма значительную величину. Однако показатель валового экспорта не позволяет выявить, какая часть стоимости китайского экспорта создается китайскими производителями, а какая ввозится из-за рубежа в виде промежуточных товаров. Чтобы преодолеть это затруднение, необходимо оценить китайский экспорт не только и не столько с точки зрения валовых показателей, а с точки зрения показателей добавленной стоимости.

Цель данной работы состоит в том, чтобы оценить особенности участия Китая в ГЦС по сравнению с крупнейшими мировыми экспортерами, такими как США и Германия.

\section{Декомпозиция экспорта в рамках ГЦС: пример Китая}

Как известно, под цепочкой добавленной стоимости (региональной или глобальной) принято понимать последовательность видов деятельности, через которые проходит производство товаров и услуг, причем различные виды деятельности могут располагаться в различных географических локациях, включая разные страны и регионы. В каждой географической локации создается определенная доля добавленной стоимости, часть которой потребляется внутри нее, а часть экспортируется. С помощью этой концепции создания стоимости мы можем измерить участие страны в цепочках добавленной стоимости.

В рамках данного подхода (Hummels, Ishii, Kei-Mu, 2001; Hummels, Rapoport, Yi, 1998; Koopman, Wang, Wei, 2011) [2, 3] стоимость национального экспорта состоит из следующих укрупненных компонентов:

1) внутренняя добавленная стоимость - ВДС (Domestic Value Added DVA), то есть стоимость, создаваемая внутри страны национальными производителями и являющаяся часть ВВП страны;

2) зарубежная добавленная стоимость - ЗДС (Foreign Value Added - FVA), то есть стоимость, создаваемая зарубежными производителями и импортируемая из-за рубежа.

Важнейшим компонентом ВДС является та ее часть, которая включается в экспорт третьих стран, то есть непосредственно продвигается по цепочке стоимости, а не потребляется в качестве финального продукта. Часто этот компонент называют показателем непрямого экспорта внутренней добавленной стоимости (Indirect Domestic Value Added - DVX).

Отсюда мы можем вывести и коэффициент, используемый для оценки степени участия страны в ГЦС (GVC Participation Index). В процентном исчислении он равняется сумме ЗДС и непрямого экспорта ВДС (FVA+DVX) [4].

Итак, проводя декомпозицию экспорта (разбивая валовый объем экспорта по источникам добавленной стоимости), мы можем измерить реальный вклад стран в мировое производство товаров и услуг. Применим данный метод декомпозиции к Китаю и выделим составляющие китайского экспорта на данных 2018 г. (рис. 3). В качестве статистики используем базу данных ОЭСР «Торговля добавленной стоимостью» (TiVA - Trade in Value Added).

Валовая стоимость китайского экспорта составила в 2018 г. 2,2 трлн долл. США, из которых 13\% (290 млрд долл. США) приходилось на зарубежную добавленную стоимость (в первую очередь, импорт полупроводников и аналогичный импорт), а 87\% (1,9 трлн долл. США) - на внутреннюю добавленную стоимость (в первую очередь, стоимость сборки в Китае товаров финального и промежуточного спроса, предназначенных для экспорта). 


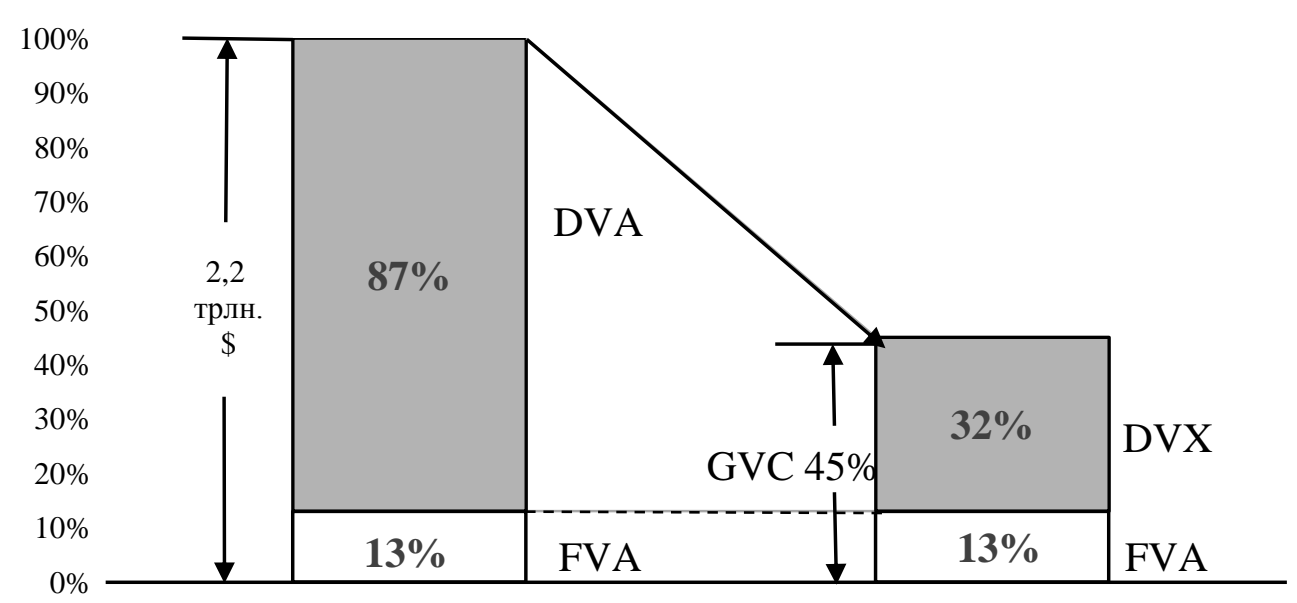

Puc. 3. Декомпозиция валового экспорта Китая в показателях добавленной стоимости, $2018(\%)$

Источник: рассчитано по данным статистической базы ОЭСР TiVA

Внутренняя добавленная стоимость (DVA) китайского экспорта распределилась в 2018 г. следующим образом: 700 млрд долл. США (что составило $32 \%$ от валового экспорта в 2,2 трлн долл. США) было использовано в качестве промежуточного продукта для экспорта из третьих стран (DVX). При сложении процентных долей FVA (13\%) и DVX (32\%) мы получаем коэффициент интеграции Китая в ГЦС, который составил в 2018 г. 45\%. Это означает, что 45\% всего экспорта Китая задействовано в глобальных цепочках создания стоимости.

Сравним теперь этот показатель с общемировыми значениями (рис. 4) и показателями отдельных стран (рис. 5-8), в частности США и Германии, которые были выбраны, поскольку наряду с Китаем они являются крупнейшими мировыми экспортерами.

На основе расчетов по данным ОЭСР (TiVA) [5], мы приходим к выводу, что общемировой коэффициент интеграции в ГЦС в течение различных периодов имел разнонаправленную динамику (рис. 4).

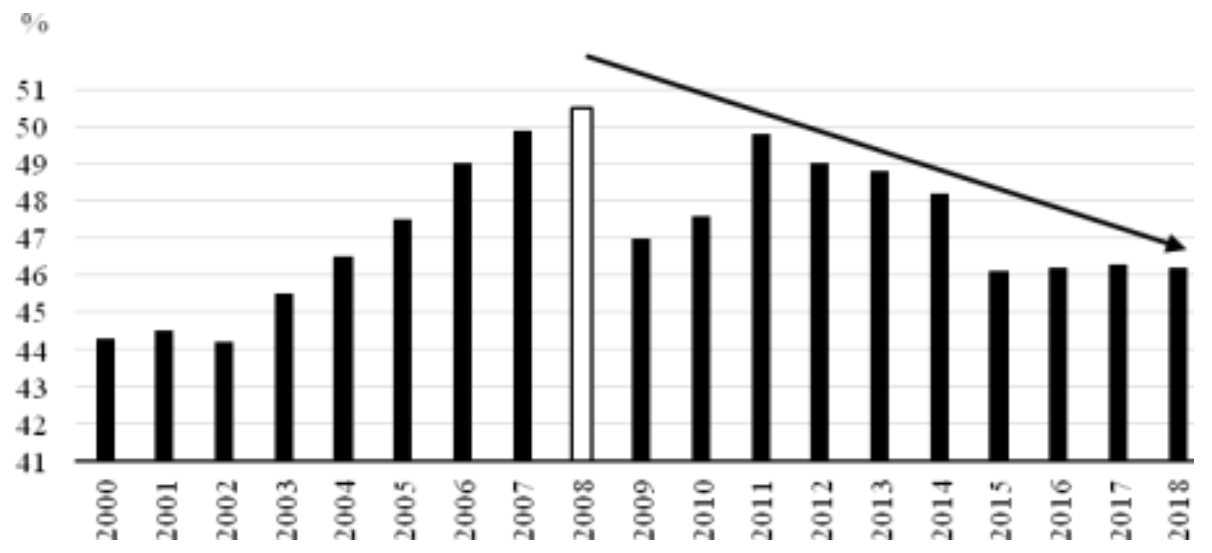

Puc. 4. Коэффициент интеграции в ГЦС: общемировой показатель, (\%) Источник: рассчитано по данным статистической базы ОЭСР TiVA

В период с 2000 по 2008 гг. процесс интеграции стран в ГЦС постепенно нарастал, отражая расширение и углубление экономической глобализации. Од- 
нако кризис 2008 г. повернул вспять эту тенденцию. Впоследствии процесс интеграции несколько укрепился, а понижательная тенденция замедлилась. В последние несколько лет коэффициент интеграции в ГЦС стабилизировался на уровне 45-46\%, достигнув уровня так называемого «плато». Интересно отметить, что начало роста коэффициента участия стран в ГЦС (общемировой показатель) совпал с вступлением Китая в ВТО в 2001 г., а начало понижательного тренда в 2008 г. сопровождалось ростом доли Китая в мировом экспорте (рис. 1).

Попробуем оценить специфику интеграции Китая в ГЦС по сравнению с крупнейшими мировыми экспортерами - США и Германией.

\section{Интеграция Китая в глобальные цепочки создания стоимости}

Как следует из методики декомпозиции валового экспорта, сокращение интеграции страны в ГЦС может происходить либо за счет снижения доли непрямой внутренней добавленной стоимости (DVX), либо за счет снижения доли зарубежной добавленной стоимости (FVA).

Сравним показатели коэффициентов интеграции США, Китая и Германии в ГЦС. Как следует из данных рис 5., на протяжении 2014-2018 гг. Китай имел наименьший коэффициент интеграции в ГЦС (около 45\%), по сравнению с Германией (около 60\%) и США (около 50\%).

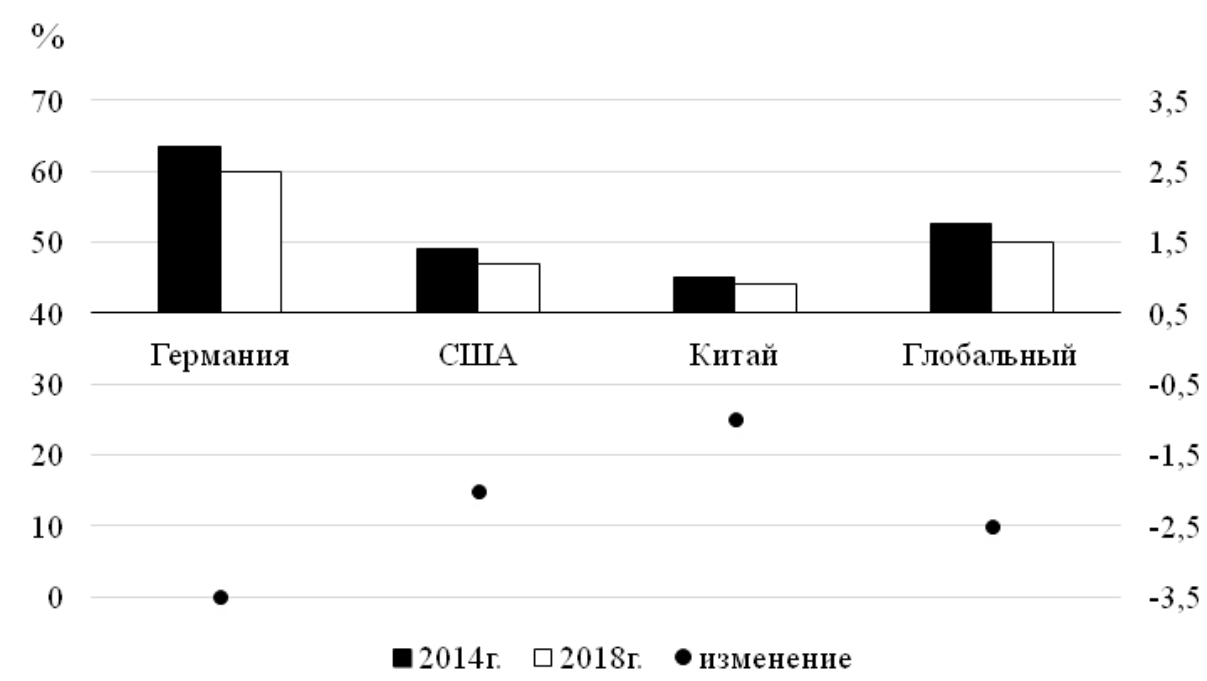

Puc. 5. Коэффициент интеграции стран в ГЦС

Примечание: коэффициент в \% - левая вертикальная ось, 2018 г.; изменение коэффициента - правая вертикальная ось, 2014-2018 гг.

Источник: рассчитано по данным статистической базы OЭСР TiVA

Изменение участия в ГЦС является понижательным как для всех стран в целом (общемировой показатель), так и для США, Германии и Китая по отдельности (фиолетовые точки, правая вертикальная ось). При этом Германия испытала наибольший спад (около 3,5\%), а Китай - минимальный (около 1\%). Объяснения такой ситуации могут быть следующие. Падение коэффициента интеграции Германии в ГЦС «стартовало» с весьма высоких первоначально достигнутых результатов. Из-за низкой первоначальной базы данного коэффициента Китае снижение, по-видимому, было меньше, чем в Германии.

Далее, рассмотрим, каким образом снижение участия каждой из рассматриваемых стран в ГЦС с динамикой внутренней добавленной стоимости (DVA). Данные рис. 6 свидетельствуют о том, что только у Китая за рассматриваемый период 
не произошло снижения ВДС, у других стран оно наблюдалось. В этом - особая специфика участия Китая в ГЦС в последние несколько лет после финансового кризиса 2008 г. О причинах такого положения дел мы скажем несколько позже.

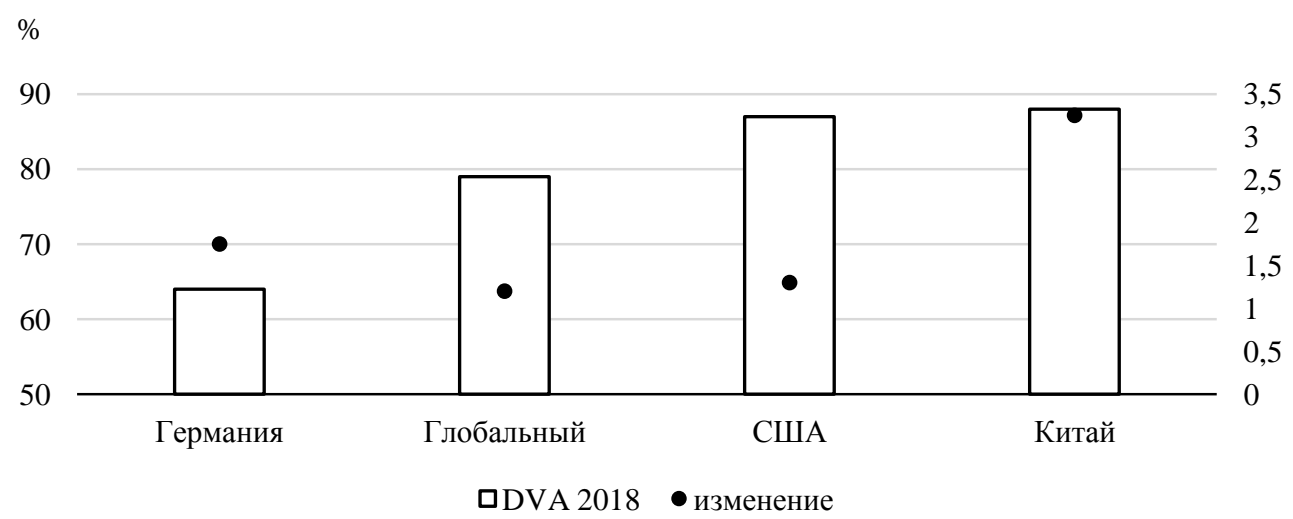

Puc. 6. Внутренняя добавленная стоимость (DVA) в страновом экспорте Примечание: доля в \% - левая вертикальная ось, 2018 г.; изменение доли - правая вертикальная ось, 2014-2018 гг.)

Источник: рассчитано по данным статистической базы ОЭСР TiVA

Что же касается зарубежной добавленной стоимости (рис. 7), то в Китае наблюдается наиболее существенное сокращение ЗДС (около 3,5\%) по сравнению с Германией (около 2,0\%) и США (около 1,0\%). Такой результат вполне ожидаем, принимая во внимание рост ВДС Китая. Поскольку, как мы упоминали выше, сумма ВДС + ЗДС = 1 (в процентном исчислении).

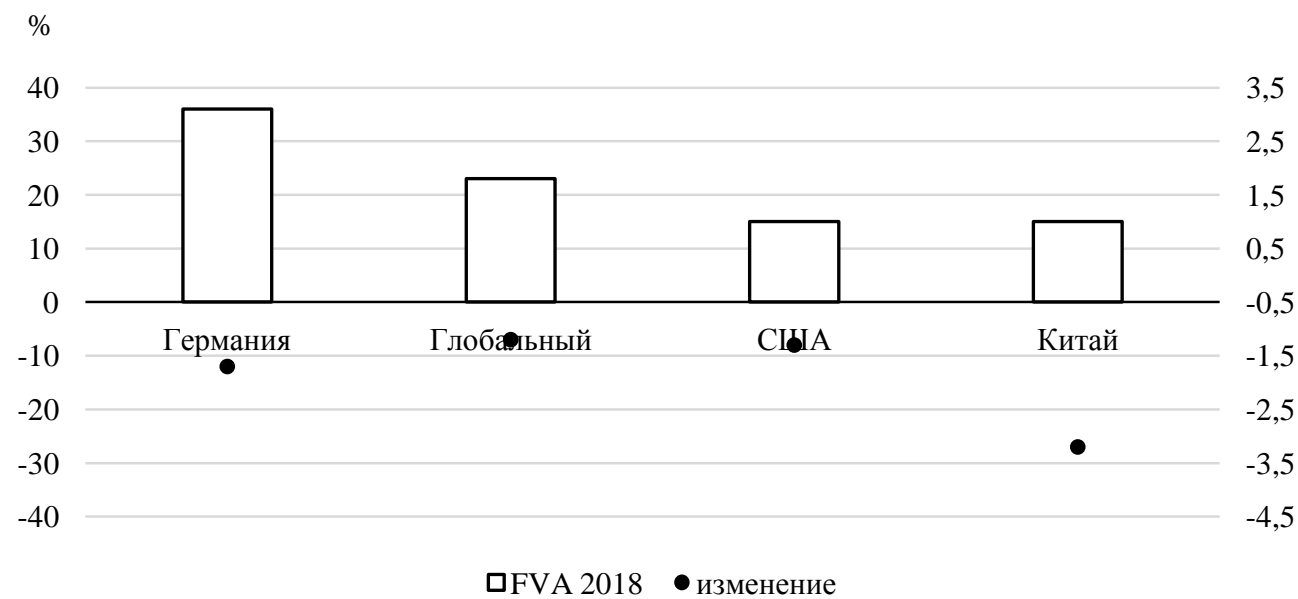

Puc. 7. Зарубежная добавленная стоимость (FVA) в страновом экспорте Примечание: доля в \% - левая вертикальная ось, 2018 г.; изменение доли - правая вертикальная ось, период 2014-2018 гг.

Источник: рассчитано по данным статистической базы ОЭСР TiVA

Снижение доли ЗДС в китайском экспорте означает, что страна имеет более низкую по сравнению с США и Германией зависимость от зарубежного промежуточного импорта и больше полагается на создание собственной внутренней стоимости в формируемой цепочке поставок.

Итак, мы объяснили, каким фактором может быть обусловлено снижение коэффициента интеграции Китая в ГЦС, которое наблюдается в последние годы. 
Теперь необходимо объяснить, почему это снижение было минимальным по сравнению с крупнейшими мировыми экспортерами, такими как США и Германия (рис. 5). Для этого необходимо проанализировать показатель непрямого экспорта внутренней добавленной стоимости.

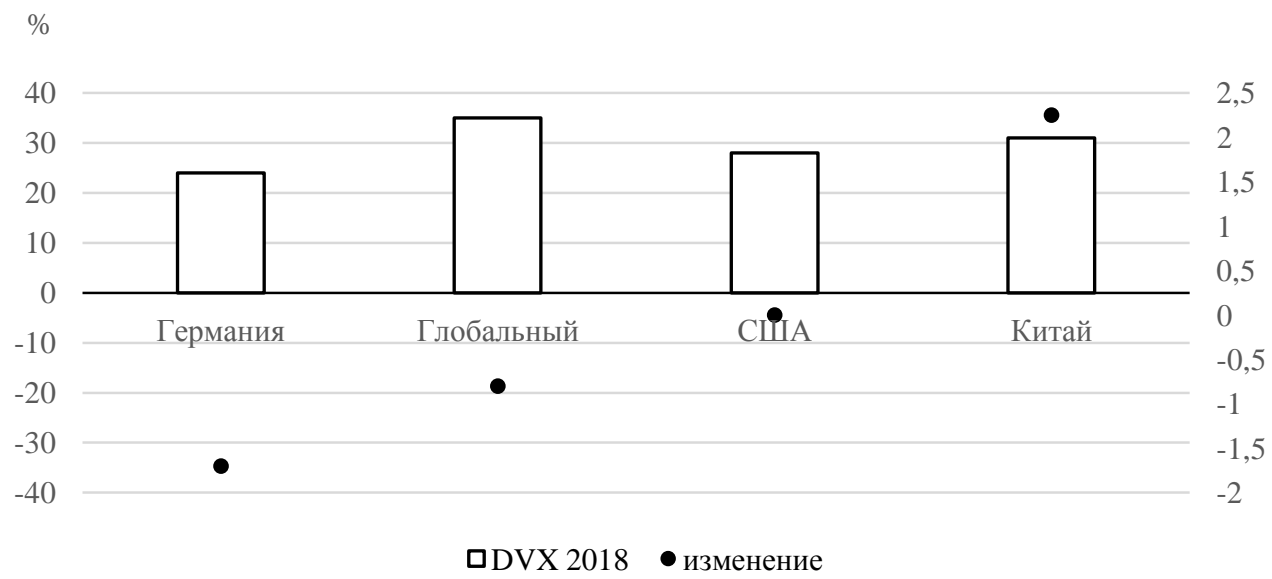

Puc. 8. Непрямая внутренняя добавленная стоимость (DVX) в страновом экспорте

Примечание: доля в \% - левая вертикальная ось, 2018 г.; изменение доли - правая вертикальная ось, период 2014-2018 гг.

Источник: рассчитано по данным статистической базы ОЭСР TiVA

Как следует из данных рис. 8, особенностью Китая является повышение этого компонента внутренней добавленной стоимости на фоне понижательных трендов для других стран и всего мира в целом. Так, снижение участия Германии в ГЦС было главным образом вызвано резким снижением DVX. То же самое относится и к США, хотя снижение и было значительно меньшим, чем в Германии.

\section{Заключение}

Суммируем некоторые выводы, вытекающие из наших расчетов.

Во-первых, для всех стран было характерно снижение интеграции в ГЦС, начало которому положил финансовый кризис 2008 г., а не американо-китайская торговая война. При этом, снижение темпов интеграции в ГЦС у Китая было минимальным, по сравнению с США, Германией и миром в целом.

Во-вторых, сокращение участия стран в ГЦС напрямую связано с уменьшением зависимости основных экономик от импортируемых промежуточных продуктов. При этом зависимость Китая от импортируемых промежуточных продуктов снижается наиболее быстрыми темпами по сравнению с крупнейшими мировыми экспортерами.

В-третьих, быстро сокращая долю зарубежной добавленной стоимости в экспорте, Китай увеличивает долю внутренней добавленной стоимости в своем экспорте, и что, особенно важно - долю непрямой внутренней добавленной стоимости, то есть резко увеличивая внутреннюю добавленную стоимость собственного экспорта промежуточных товаров, направляемых в третьи страны (DVX). Это свидетельствует о том, что Китай не только получает более высокие экспортные доходы, но и целенаправленно формирует свою собственную цепочку поставок, наращивая внутренние производственные мощности.

Увеличение ВДС происходит не в последнюю очередь в связи с запуском национальной стратегии «Сделано в Китае до 2025 года». в которой определены 
ключевые отрасли обрабатывающей промышленности, которые Китай будет развивать для уменьшения своей зависимости от других развитых регионов мира; в рамках подхода ГЦС - это уменьшение доли ЗДС в китайском экспорте.

Данная стратегия была неоднозначно воспринята во многих развитых странах. США во главе с Трампом являются ярким примером такого отношения. Так, США обвинили Китай в росте меркантилизма (протекционизма) и предприняли ряд ответных торговых мер, не ограничиваясь введением тарифов на китайские товары. И хотя Китай впоследствии скорректировал свои стратегические амбиции, он попрежнему продолжает работать в направлении повышения внутренней добавленной стоимости своего экспорта (особенно в ключевых отраслях промышленности).

И наконец, можно ли говорить об экономической деглобализации на основе данных о снижении участия стран в ГЦС, которое мы показали на общемировых показателях, а также на показателях крупнейших экономик мира - США, Германии и Китая. Некоторые эксперты утверждают, что рост протекционизма, торговые войны, безусловно, свидетельствуют о трендах деглобализации мировой экономики. Мы придерживаемся более мягкой позиции. Последние данные о динамике внутренней и зарубежной добавленной стоимости в ГЦС свидетельствуют о том, несмотря на то, что участие стран в ГЦС снижается, оно остается еще весьма высоким, и говорить об устоявшихся трендах деглобализации пока рано. С точки зрения подхода ГЦС, мы пока наблюдаем снижение темпов экономической глобализации, которые были усилены последствиями пандемии коронавируса COVID-19.

\section{Список источников / References}

1. World Trade Organization. Available at: https://timeseries.wto.org/ (accessed 20.04.2020).

2. Hummels D.L., Ishii Jun, Kei-Mu Yi. The Nature and Growth of Vertical Specialisation in World Trade. Journal of International Economics, 2001, vol. 54. no. 1, pp.75-96. DOI:10.1016/S0022-1996(00)00093-3

3. Hummels D.L., Rapoport D., Yi Kei-Mu. Vertical Specialization and the Changing Nature of World Trade. Economic Policy Review, 1998, vol. 4. no. 2, pp.79-99.

4. Koopman R., Wang Z., Wei S.-J. Tracing value-added and double counting in gross exports. American Economic Review, 2011, vol. 104. no. 2, pp. 459-494. DOI: 10.1257/aer.104.2.459

5. World Input-Output Databast. Available at: http://www.wiod.org/otherdb\#ADB (accessed 20.04.2020).

\section{Сведения об авторах / About authors}

Волгина Наталья Анатольевна, доктор экономических наук, профессор кафедры международных экономических отношений, Экономический факультет, Российский университет дружбы народов. 117198 Россия, Москва, ул. Миклухо-Маклая, 6., каб. 104. E-mail: volgina_na@rudn.ru Natalia A. Volgina, Doctor of Economic Sciences, Professor of the Department of International Economic Relations, Faculty of Economics. Peoples' Friendship University of Russia. 6, Mikhluho-Maklaya Str., Office 104, Moscow, Russia 117198.E-mail:volgina_na@rudn.ru

Лю Пэнфэй, аспирант кафедры международных экономических отношений, Российский университет дружбы народов. 117198 Россия, Москва, ул. Миклухо-Маклая, 6, каб. 104.

E-mail: lynvb@yandex.ru

Liu Peng-fei, PhD student, Department of International Economic Relations, Faculty of Economics, Peoples' Friendship University of Russia. 6, Miklukho-Maklaya str., Office 104, Moscow, Russia 117198. E-mail:lynvb@yandex.ru 